\title{
Numerical analysis of air effect on the powder flow dynamics in the FT4 Powder Rheometer
}

\author{
Wenguang Nan ${ }^{1,2}$, Vincenzino Vivacqua ${ }^{1}$, Mojtaba Ghadiri ${ }^{1 *}$, and Yueshe Wang $^{2}$ \\ ${ }^{1}$ Institute of Particle Science and Engineering, University of Leeds, Leeds LS2 9JT, UK \\ ${ }^{2}$ State Key Laboratory of Multiphase Flow in Power Engineering, Xi'an Jiaotong University, Xi' an 710049, China
}

\begin{abstract}
The FT4 powder rheometer of Freeman Technology is widely used nowadays in industry for characterisation of particle flow under dynamic conditions of shear strain rate. It measures the work (termed flow energy) required to penetrate a rotating impeller into a powder bed. However, little is known about its underlying powder mechanics, i.e. the relationship between the flow energy and the prevailing local shear stress. This has recently been studied, but only for very simple and ideal systems amenable to analysis by DEM. We analyse the effect of gas flow through the powder bed on the flow behaviour of cohesionless particles in FT4 by DEM-CFD simulation. The results show that the relative particle velocities induced by the mean shear speed, is of the same order as that produced by the root of granular temperature. The shear stress in both cases with and without gas flow could be quantified by the inertial number. The flow energy correlates well with the shear stress in front of the blade, and both increase with the inertial number and could be significantly reduced by the upward gas flow.
\end{abstract}

\section{Introduction}

The study of powder flow as a function of strain rate is of great interest in fast feeding, conveying, mixing and packaging processes. Conventional devices for assessing the flowability of powders, such as shear cell $[1,2]$, usually operate at low levels of shear strain rate, i.e. in the quasi-static flow regime. However, in many processes the powder is subjected to higher values of strain rate, and the characterisation of the flow behaviour needs to be carried out in dynamic conditions as in a Couette device $[3,4]$ in order to obtain meaningful measurements.

Recently, the FT4 powder rheometer of Freeman Technology (Tewkesbury, UK) has received attention for its capability to operate in the dynamic conditions, where a twisted blade rotates at a set tip speed whilst translating in or out of the powder bed [5]. The input work required to drive the rotating blade into the powder bed, termed 'flow energy', is measured and advocated to represent the ease with which powder flows. This instrument is nowadays widely used for powder rheometry in industry and academia [6-8]. Several studies have focused on the analysis of the powder mechanics of FT4 by numerical simulations. Bharadwaj et al. [9] used the Discrete Element Method (DEM) to simulate the effect of friction coefficient and particle shape on the particle flow in FT4, and showed that the flow energy was sensitive to the particle shape and friction coefficients. Hare et al. [10] simulated the dynamics of FT4 powder rheometer for a bed of glass beads made cohesive by silanisation and showed that the shear stress increased with the penetration depth. To evaluate the effect of aeration on the flow properties, a controlled gas flow (usually air) is optionally introduced into the particle bed through a porous stainless steel disc at the base of the vessel. As a result, the blade rotating and moving down into the bed will encounter less resistance and the measured flow energy will decrease. However, the effect of gas flow on the particle flow at high strain rates in FT4 rheometer has not so far been analysed.

We report on our numerical analysis of the dynamic behaviour of the particle bed under aeration conditions by DEM-CFD simulation. By varying the permeating air velocity and impeller tip speed, the effect of air drag on the particle flow under different strain rates is analysed. This is of particular relevance to fine and low density particles, as their motion is affected by air drag. Besides the flow energy, the stresses within the powder bed immediately in front of the blade are also analysed. It provides a step towards understanding the dynamics of the FT4 powder rheometer in the presence of air.

\section{Simulation methods and conditions}

The FT4 powder rheometer used in this work comprises a cylindrical glass vessel with $25 \mathrm{~mm}$ diameter and a corresponding $23.5 \mathrm{~mm}$ diameter stainless steel blade, as shown in Fig. 1(a) and (b). As the initial packed bed is almost uniform, only the downward test is considered to reduce the computational time, where the blade rotates anti-clockwise and moves into the powder bed while pressing down. In this way, the blade action is more

Corresponding author: M.Ghadiri@,leeds.ac.uk 
compacting than slicing, resulting in shearing the bed, whilst applying normal stresses to the particle bed by the blade. The variation of the vertical translational velocity $u$ and rotational velocity $\omega$ with the tip speed $u_{\text {tip }}$ used in the simulation is shown in Table 1, where a helix angle of $-5^{\circ}$ is used:

Table 1. Values of the translation and rotational velocity.

\begin{tabular}{|c|c|c|c|c|c|c|}
\hline$u_{\text {tip }}, \mathrm{m} / \mathrm{s}$ & 0.025 & 0.05 & 0.10 & 0.25 & 0.50 & 1.00 \\
\hline$u, \mathrm{~mm} / \mathrm{s}$ & 2.18 & 4.36 & 8.72 & 21.8 & 43.6 & 87.2 \\
\hline$\omega, \mathrm{rad} / \mathrm{s}$ & 2.12 & 4.24 & 8.48 & 21.2 & 42.4 & 84.8 \\
\hline
\end{tabular}

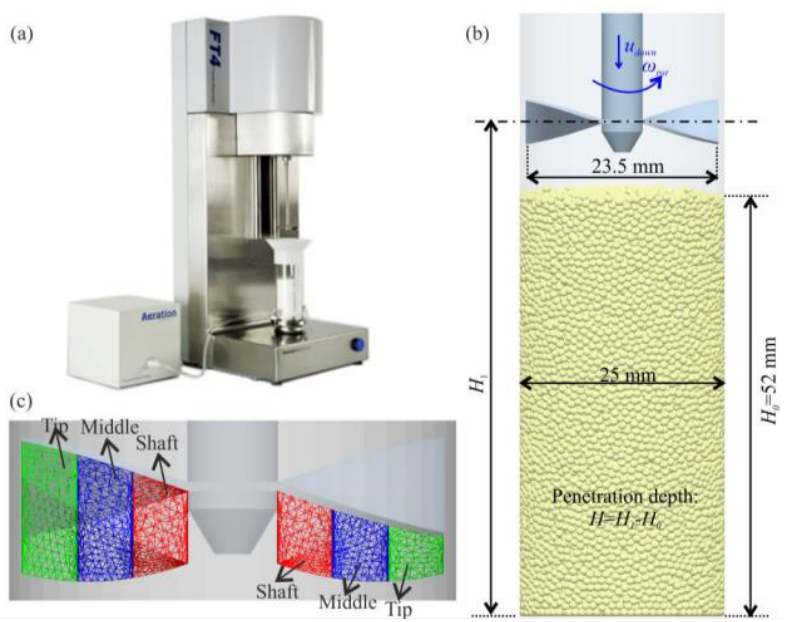

Fig. 1. Schematic illustration of (a) basic set up, (b) simulation system and (c) measured cells.

This process is simulated by DEM-CFD, where particles are modelled as discrete elements and their motions are tracked individually by solving Newton's laws of motion, whilst the fluid phase is continuum. Using the poured packing method, 36,000 spheres with a flat distribution of diameters in the size range of 0.85-1.0 $\mathrm{mm}$ are generated to form a packed bed with height of $52 \mathrm{~mm}$ and porosity of 0.41 . The material properties and interaction parameters are listed in Table 2 and Table 3, respectively. For DEM-CFD simulations, air with density of $1.2 \mathrm{~kg} / \mathrm{m}^{3}$ and viscosity of $1.8 \times 10^{-5} \mathrm{~Pa} \cdot \mathrm{s}$ is used. The gas flow is introduced from the bottom of the vessel and three superficial gas velocities are simulated, $0.1,0.2$ and $0.3 \mathrm{~m} / \mathrm{s}$. The effect of blade motion on the air flow is considered by using the method of dynamic mesh. For the case with no gas flow, only DEM simulation is used, as the particles are large and the interstitial fluid drag is negligible.

Table 2. Material properties in simulations.

\begin{tabular}{|c|c|c|c|}
\hline Material property & Particles & $\begin{array}{c}\text { Impeller } \\
\text { blade }\end{array}$ & $\begin{array}{c}\text { Cylinder } \\
\text { vessel }\end{array}$ \\
\hline Density, $\rho\left(\mathrm{kg} / \mathrm{m}^{3}\right)$ & 1000 & 7800 & 2500 \\
\hline Shear modulus, $G(\mathrm{~Pa})$ & $1 \times 10^{8}$ & $7.3 \times 10^{10}$ & $2.4 \times 10^{10}$ \\
\hline Poisson's ratio, $v$ & 0.35 & 0.3 & 0.3 \\
\hline
\end{tabular}

Table 3. Contact interaction parameters in simulations.

\begin{tabular}{|c|c|c|}
\hline Interaction property & $\begin{array}{c}\text { Particle- } \\
\text { particle }\end{array}$ & $\begin{array}{c}\text { Particle- } \\
\text { blade/vessel }\end{array}$ \\
\hline Friction coefficient, $\mu$ & 0.5 & 0.25 \\
\hline Restitution coefficient, $e$ & 0.6 & 0.6 \\
\hline
\end{tabular}

\section{Results and discussions}

Snapshots of the simulated flow pattern are shown in Fig. 2. For small penetration depth as shown in Fig. 2(a) and (b), the free surface changes with time as the blade moves. As the blade is driven down further, the free surface of the particle bed becomes flat when the penetration depth is larger than $20 \mathrm{~mm}$, as shown Fig. 2(c) and (d). The impeller motion mainly affects the particles locally around the blade, whilst particles remote from the impeller are essentially stationary. These results suggest that the particle flow in front of the blade can be representative of particle behaviour. The measurement cells in front of the blade spans the width of the blade as also adopted by Hare et al. [10] are used, as shown in Fig. 1(c). The cell-averaged granular temperature [11] of particles $\theta$ is normalised by the strain rate $\gamma$, which is calculated by the particle velocity gradient, given as:

$$
\begin{gathered}
\theta=\sqrt{\frac{1}{3 N} \sum_{p=1}^{N}\left[\left(u_{p}-u_{a v g}\right)^{2}+\left(v_{p}-v_{a v g}\right)^{2}+\left(w_{p}-w_{a v g}\right)^{2}\right]} \\
S=\frac{\gamma d}{\theta}
\end{gathered}
$$

where $N$ is the particle number in the considered measurement cell; $u_{p}, v_{p}$ and $w_{p}$ are the velocity components of particle $p$ in $x, y$ and $z$ directions, respectively; $u_{\text {avg }}, v_{\text {avg }}$ and $w_{\text {avg }}$ are the corresponding mean value of $u_{p}, v_{p}$ and $w_{p}$, respetively; $d$ is the particle diameter. The strain rate is only determined by the impeller tip speed and is not sensitive to the gas flow rate. The variation of the non-dimensional granular temperature with the tip speed and gas flow rate is shown in Fig. 3. The gas flow rate does not have much influence on the granular temperature of particles. $S$ is almost constant (around 2.0) and shows a little decrease when the strain rate is low. It suggests that the relative particle velocities induced by the mean shear speed $d \gamma$, is of the same order as that produced by the granular temperature $\theta$.

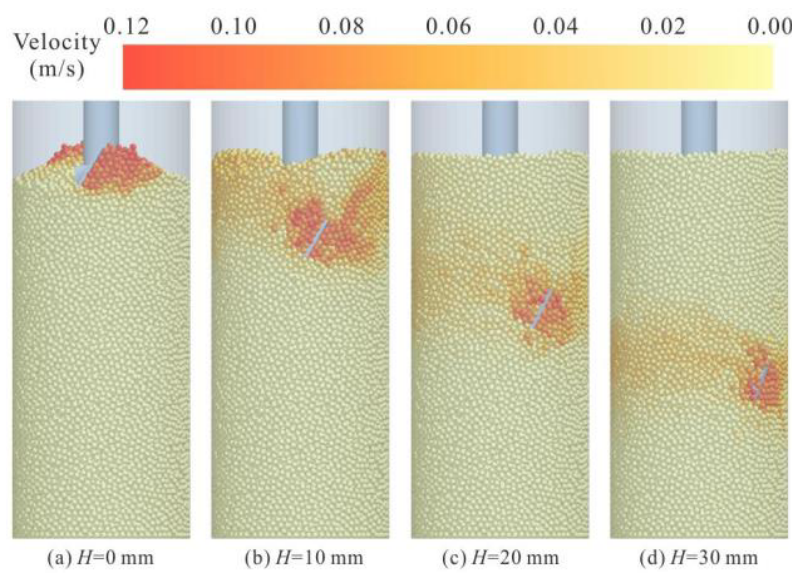

Fig. 2. The simulated flow pattern of particle flow $\left(u_{t i p}=0.1 \mathrm{~m} / \mathrm{s}\right.$ and $u_{f}=0.0 \mathrm{~m} / \mathrm{s}$ ). 


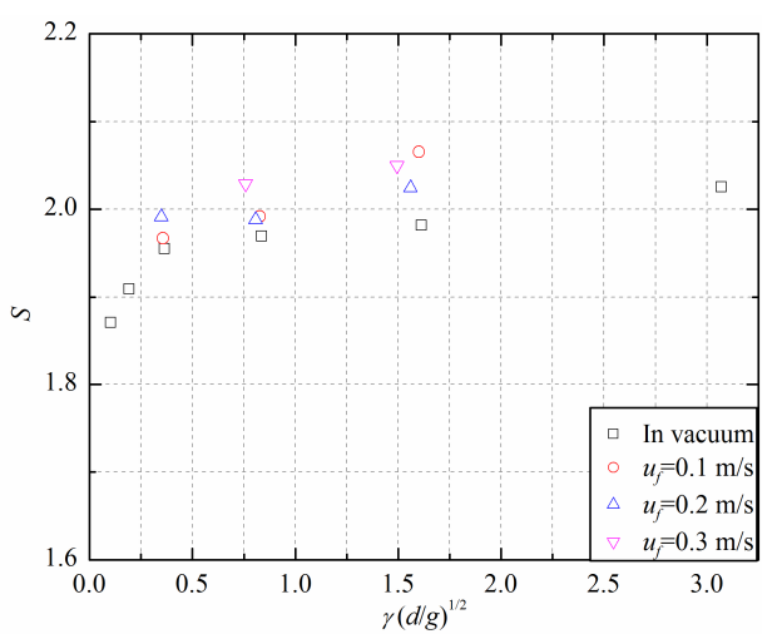

Fig. 3. The variation of non-dimensional granular temperature.

\subsection{Stress analysis}

The stress of particle flow is also analysed immediately in front of the blade, and the stress tensor is given by:

$$
\sigma_{i j}=\frac{1}{V}\left(\sum_{p \in V} m_{p} \delta v_{i} \delta v_{j}+\sum_{c \in V} f_{i j} \cdot r_{i j}\right)
$$

where $V$ is the cell volume; $m_{p}$ is the mass of particle $p$; $\delta v_{i}$ and $\delta v_{j}$ are the fluctuation velocities of particle $p ; f_{i j}$ is the contact force at contact $c$ and $r_{i j}$ is the corresponding branch vector. Based on the stress tensor, three principal stresses are calculated: major one $\sigma_{1}$, intermediate one $\sigma_{2}$ and minor one $\sigma_{3}$. The normal stress $p$ and shear stress $\tau$ are then given as:

$$
\begin{gathered}
p=\frac{\sigma_{1}+\sigma_{2}+\sigma_{3}}{3} \\
\tau=\frac{\sqrt{\left(\sigma_{1}-\sigma_{2}\right)^{2}+\left(\sigma_{1}-\sigma_{3}\right)^{2}+\left(\sigma_{2}-\sigma_{3}\right)^{2}}}{\sqrt{6}}
\end{gathered}
$$

Similar to the characterisation of the flow regime, the inertial number $I[11]$ is also used, given as:

$$
I=\gamma d \sqrt{\rho_{p} / p}
$$

The inertial number is interpreted as the ratio between the inertial timescale $d /\left(p / \rho_{p}\right)^{0.5}$ and the macroscopic deformation timescale $(1 / \gamma)$. It follows that the inertial number decreases with the blade penetration depth due to the increased normal stress.

The variation of the shear stress with the inertial number is shown in Fig. 4. The shear stress decreases with the inertial number, in the form of negative power law. The two cases with air flow and without accounting for its effect (i.e. vacuum) both show the same trend and collapses to one curve. It suggests that the evolution of the shear stress could be described by the inertial number. To further depict this mechanism, the "viscosity" $\tau / \gamma$ (the resistance to deformation rate by the shear stress) under different tip speeds is shown in Fig. 5, where the cases with air flow are not included for simplicity as they have the same trend with the cases without air flow. It is interesting to note that $\ln (\tau / \gamma)$ shows a linear decrease with $\ln (I)$ in all cases. The "viscosity" could be described by the following equation:

$$
\tau / \gamma=a+b I^{-n}
$$

where $a, b$ and $c$ are parameters which vary with the tip speed. Their value calculated by the least square fitting is shown in Table 4. $n$ is almost 2.0, indicating that $\tau \sim I^{-2}$. With the increase of the tip speed, $b$ increases whilst $a$ decreases, suggesting that the dependence of $\tau$ on $I$ is stronger for larger tip speed.

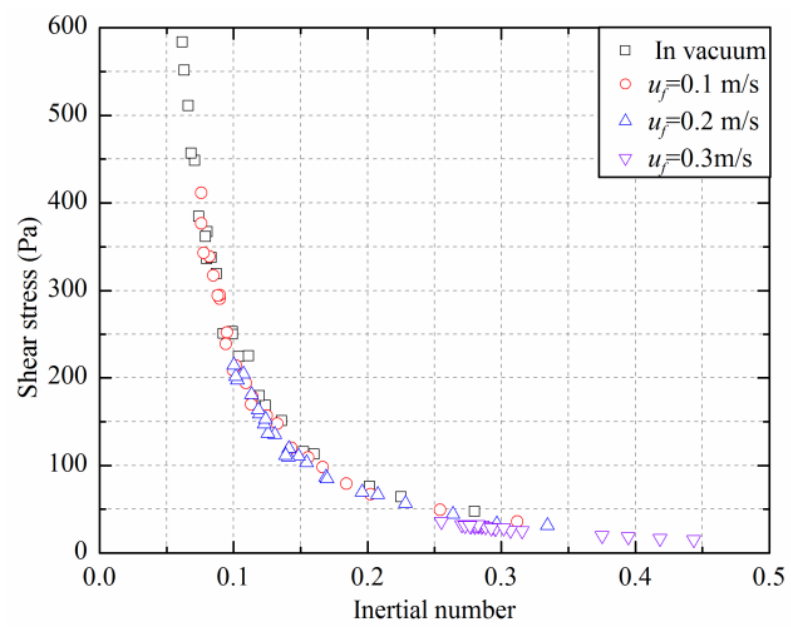

Fig. 4. The evolution of the shear stress with inertial number.

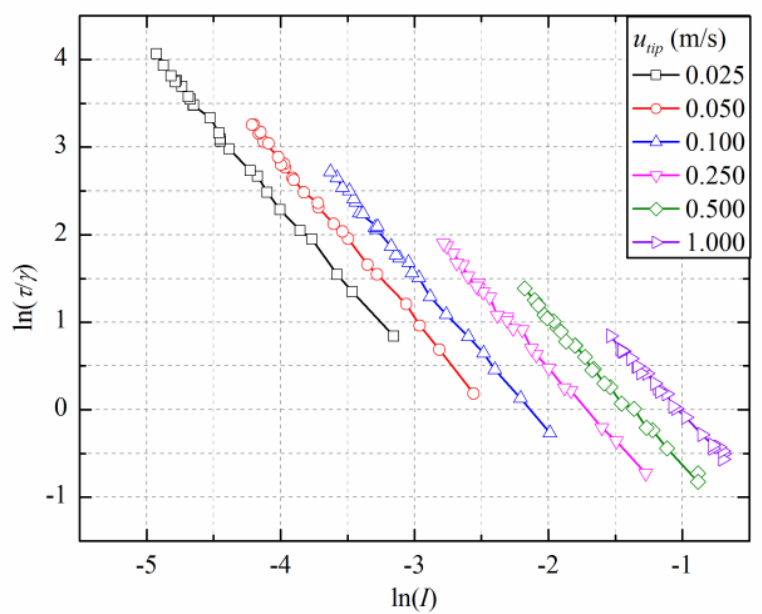

Fig. 5. Variation of the viscosity with inertial number.

Table 4. The values of the fitting parameters.

\begin{tabular}{|c|c|c|c|c|c|c|}
\hline$u_{\text {tip }}$ & 0.025 & 0.05 & 0.1 & 0.25 & 0.5 & 1.0 \\
\hline$a$ & 1.269 & 0.664 & 0.194 & 0.171 & 0.158 & 0.079 \\
\hline$b$ & 0.0026 & 0.0054 & 0.0135 & 0.0301 & 0.0575 & 0.1558 \\
\hline$n$ & 2.024 & 2.008 & 1.933 & 1.933 & 1.929 & 1.727 \\
\hline
\end{tabular}

\subsection{Flow energy}

The total input work $E$, which is also referred to as 'flow energy' of the powder is given as:

$$
E=\int_{0}^{H}\left(\frac{T}{R \tan (\alpha)}+F\right) d H
$$


where $T$ and $F$ are the torque and axial force required for the blade motion, respectively; $R$ is the blade radius; $\alpha$ is the helix angle; $H=H_{1}-H_{0}$ is the penetration depth as shown in Fig. 1(b), where $H_{1}$ is the average vertical position of the blade and $H_{0}$ is the initial bed height. The flow energy is normalised by the potential energy of particles $\left(m_{b} g H\right.$, where $m_{b}$ is the total particle mas above the blade) and then averaged for the last $20 \mathrm{~mm}$ depth.

The variation of non-dimensional flow energy with the inertial number is shown in Fig. 6. At very low inertial numbers, the flow energy is almost constant as the particle flow is in the quasi-static regime. At large inertial numbers, the flow energy increases with the inertial number in the form of power law. The shear stress (normalised by dividing it by $m_{b} g / A$, where $A$ is the cross-section area of the vessel) shows a similar trend and is not shown here for simplicity. The results clearly show that there exists a direct relationship between the flow energy and shear stress, as shown in Fig. 7. The flow energy increases with the shear stress in a linear trend for both cases of vacuum and gas flow. In summary, the flow energy as determined by FT4 is well correlated with the shear stress. The latter is of course a function of the strain rate.

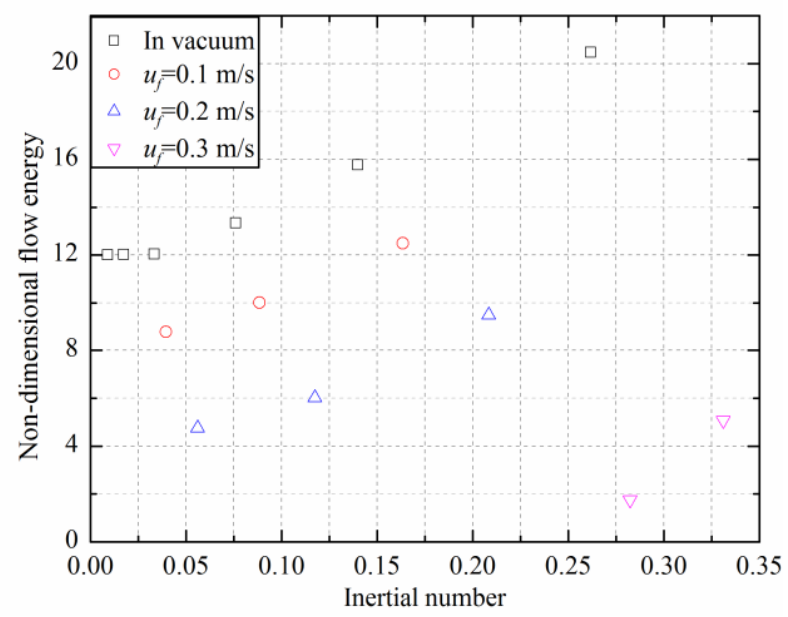

Fig. 6. Variation of the flow energy with ineritial number.

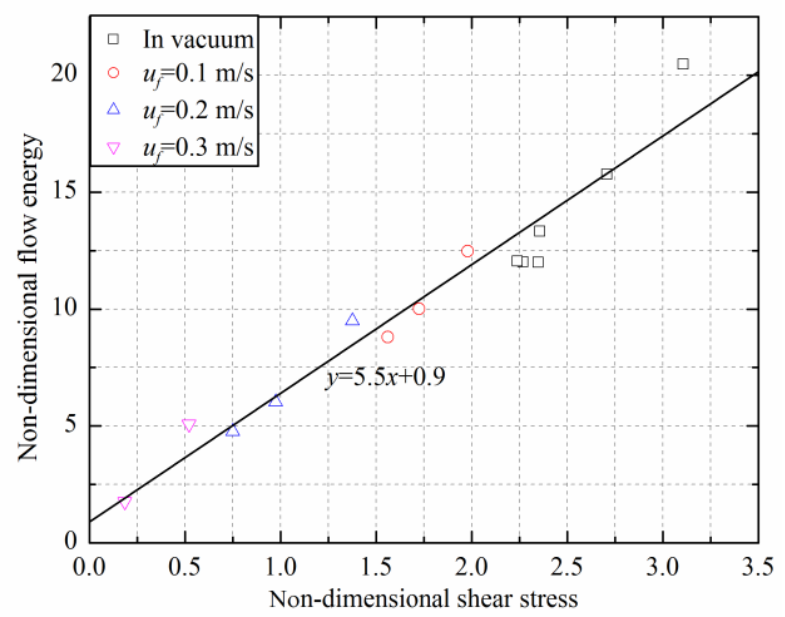

Fig. 7. The variation of flow energy with shear stress [12].

\section{Conclusions}

The rheological behaviour of spherical cohesionless particles subjected to shearing flows by an impeller in the presence of an upward gas flow has been analysed under high shear strain rates using the FT4 instrument by DEM-CFD simulations. The bulk particles are subjected to the standard downward test procedure of FT4 rheometer, where a rotating blade is driven into the particle bed. The main results from the present study are summarised as follows:

1) The relative particle velocities induced by the mean shear speed $d \gamma$, is of the same order as that produced by the granular temperature $\theta$.

2) The evolution of shear stress could be quantified by the inertial number, and an equation is proposed to quantify the effect of tip speed on the pseudo-viscosity of particle flow.

3) The flow energy and shear stress both increase with the inertial number and decrease significantly with the gas flow. The flow energy correlates well with the shear stress in front of the blade for all conditions including permeating air, implying that the flow energy could also be used to map out the flow regimes.

\section{Acknowledgments}

The authors are grateful to the scholarship from China Scholarship Council (CSC) for the first author. We are thankful to DEM Solutions, Edinburgh, UK, for providing a special license for the EDEM software for use in this work, and also to Drs Colin Hare and Mehrdad Pasha for their generous help in setting up the computational facility. We also thank Freeman Technology for making available an FT4 instrument for the experiment, as well as technical support and comments from Dr Doug Millington-Smith and Mr Tim Freeman.

\section{References}

1. J. Schwedes, Granular Matter 5, 1-43 (2003).

2. A.W. Jenike, Bulletin No. 108, Utah State University, (1961).

3. G.I. Tardos, S. McNamara, I. Talu, Powder Technology 131, 23-39 (2003).

4. M.K. Langroudi, S. Turek, A. Ouazzi, G.I. Tardos, Powder Technology 197, 91-101 (2010).

5. R. Freeman, Powder Technology 174, 25-33 (2007).

6. H. Kinnunen, G. Hebbink, H. Peters, J. Shur, R. Price, AAPS PharmSciTech 15, 898-909 (2014).

7. A. Vasilenko, B.J. Glasser, F.J. Muzzio, Powder Technology 208, 628-636 (2011).

8. J.G. Osorio, F.J. Muzzio, Drug Dev Ind Pharm 39, 1464-1475 (2013).

9. R. Bharadwaj, W.R. Ketterhagen, B.C. Hancock, Chemical Engineering Science 65, 5747-5756 (2010).

10. C. Hare, U. Zafar, M. Ghadiri, T. Freeman, J. Clayton, M.J. Murtagh, Powder Technology 285, 123-127 (2015).

11. G.D.R. MiDi, Eur Phys J E Soft Matter 14, 341365 (2004).

12. W.G. Nan, M. Ghadiri, Y.S. Wang, Cheimcal Engineering Science 162, 141-151(2017). 